Ensino, Saúde e Ambiente - V5 (3), pp. 84-93, dez. 2012

\title{
ESTÁGIO DE DOCÊNCIA EM SAÚDE DO HOMEM: UMA EXPERIÊNCIA NA ESTRATÉGIA DE SAÚDE DA FAMÍLIA
}

\section{TEACHING INTERNSHIP IN MEN'S HEALTH: AN EXPERIENCE IN FAMILY HEALTH PROGRAM}

\author{
Fabrício Almeida dos Santos ${ }^{1}$, Adriana Alves Nery ${ }^{2,}$ Vilara Maria Mendes \\ Mesquita Pires ${ }^{2}$ \\ ${ }^{1}$ Secretaria Municipal de Saúde de Aracaju, fadsantosenf@ hotmail.com \\ ${ }^{2}$ Departamento de Saúde da UESB, aanery@ gmail.com, vilaragondim@ yahoo.com.br
}

\section{RESUMO}

Relato de Experiência que objetiva apresentar a vivência na disciplina Estágio de Docência ao desenvolver atividades voltadas à Saúde do Homem numa Unidade de Saúde da Família (USF) a partir da integração com a disciplina Estágio Curricular Supervisionado I do Curso de Graduação em Enfermagem da Universidade Estadual do Sudoeste da Bahia (UESB), Campus de Jequié. Foram desenvolvidas atividades de educação em saúde para usuários da USF, oficina pedagógica sobre Saúde do Homem direcionada aos Agentes Comunitários de Saúde, discussões de artigos científicos e supervisão durante as consultas de enfermagem realizadas pelos discentes. Constatou-se que, discutir Saúde do Homem na Estratégia de Saúde da Família, foi uma experiência nova para todos os sujeitos envolvidos. Espera-se contribuir a partir deste estudo para outras vivências dessa natureza que possibilitem uma maior compreensão, por parte de profissionais de saúde, acadêmicos e comunidade, sobre a relação estabelecida do homem com o cuidado à sua saúde.

Palavras-chave: Ensino, Estratégia de Saúde da Família, Saúde do Homem.

\section{ASTRACT}

This is an Experience Report to present our experience in the discipline Internship Teaching when developing activities aimed at 'Men's Health Unit in a Family Health Unity' (USF) under the integration with the discipline 'Supervised Internship Course I' of the Undergraduate Nursing Course of the State University of the Southeast of Bahia, Campus Jequié. Education activities to the users of USF were developed, as well as educational workshop on Human Health directed to Community Health Agents, discussions on scientific papers and supervision during the consultations with the nursing students. It was found that discussing Men's Health in Family Health Strategy was a new experience for all involved. It is expected from this study to contribute to other experiences of this nature that will allow greater understanding on the part of health professionals, academics and community on the established relationship of man with the care of their health.

Key-words: Teaching, Family Health Program, Men’s Health

\section{INTRODUÇÃO}

A disciplina "Estágio de Docência" é uma disciplina de natureza optativa do Programa de Pós-Graduação em Enfermagem e Saúde, em nível de mestrado da Universidade Estadual do Sudoeste da Bahia (UESB), Campus de Jequié. Possui dois 
créditos teóricos e totaliza uma carga horária de $30 \mathrm{H}$. A ementa da disciplina objetiva a promoção do espaço de interligação docente/discente, possibilitando ao mestrando o exercício da prática acadêmica.

Visando uma aproximação com o objeto de estudo da dissertação, analisar a dinâmica da acessibilidade masculina à Estratégia de Saúde da Família, a partir de algumas discussões teóricas e práticas com a orientadora, decidiu-se desenvolver as atividades da disciplina Estágio de Docência numa Unidade de Saúde da Família (USF) no que tange a integração com a disciplina Estágio Curricular Supervisionado I (ECS I). Essa disciplina é oferecida no VIII semestre do Curso de Graduação em Enfermagem da UESB, possui carga horária de 270 horas e suas atividades são exclusivamente realizadas numa USF, sendo acompanhadas por uma enfermeira preceptora e pela docente responsável da disciplina.

Objetivou-se através do presente estudo vivenciar atividades voltadas à saúde do homem, ampliando a dimensão científica da pós-graduação, além dos saberes inerentes à compreensão do processo de trabalho sobre a temática numa Unidade de Saúde da Família.

\section{CONTEXTUALIZANDO A SAÚdE DO HOMEM SOB O ENFOQUE DA ESTRATÉGIA DE SAÚDE DA FAMÍLIA}

Modelo de assistência à saúde com foco na família, o Programa de Saúde da Família (PSF) criado no ano de 1994 foi inspirado na realidade sanitária de países como Cuba, Inglaterra, Canadá e Suécia. Apesar de ter surgido como um Programa, o PSF se configura, atualmente, como uma Estratégia ${ }^{l}$, pois possibilita um trabalho dinâmico de diagnóstico, planejamento, implementação e avaliação de ações em saúde de uma equipe multidisciplinar destinado a uma população circunscrita em um território definido.

O objetivo geral do PSF é contribuir para a reorientação do modelo assistencial a partir da atenção básica, em conformidade com os princípios do

\footnotetext{
${ }^{1}$ Utilizou-se a terminologia no estudo de Estratégia de Saúde da Família (ESF), uma vez que é desta forma o entendimento hoje colocado pelo Ministério da Saúde, considerando a Saúde da Família como uma estratégia para a reorganização da atenção básica. Em determinados momentos, referiu-se ao Programa de Saúde da Família utilizando a terminologia presente nos documentos oficiais do Ministério da Saúde brasileiro.
} 


\section{Ensino, Saúde e Ambiente - V5 (3), pp. 84-93, dez. 2012}

SUS, imprimindo uma nova dinâmica de atuação nas unidades básicas de saúde, com definição de responsabilidades entre os serviços de saúde e a população (BRASIL, 1997, p.11).

Após quase quinze anos de existência da Estratégia de Saúde da Família, a maioria das ações das equipes de saúde ainda são voltadas para a saúde da mulher e da criança, concepção esta, talvez, remanescente dos primórdios do Programa de Agentes Comunitários de Saúde (PACS). O esperado era que as ações pudessem contemplar a família em sua integralidade, respeitando sua dinâmica e particularidades no enfoque da vigilância à saúde.

Nesse contexto, o homem aparece, com frequência, como um sujeito que tivesse sido deixado em plano secundário durante todo esse processo de conformação e consolidação da ESF, mesmo estando ele presente e intensa nos indicadores de morbimortalidade que preocupam a saúde pública no país.

Por outro lado, atualmente, tem sido bastante discutida a captação de usuários do sexo masculino, inclusive através de campanhas publicitárias do Ministério da Saúde através de programas de rádio e televisão, incentivando que o homem procure as unidades de saúde no intuito de minorar uma estatística desfavorável, neste momento, ao seu processo saúde-doença.

Banks (2004) aponta alguns fatores que podem influenciar essa reduzida procura dos homens pelos serviços de saúde: a distância entre o local de trabalho e serviço de atenção primária à saúde, a "limitação dos horários de funcionamentos desses serviços", a crença do homem de ser "forte", o que gera conflito quando ele adoece e, por fim, o estereótipo que os serviços de saúde, em sua maioria, são espaços para o público materno-infantil, e não para homens.

A princípio, falar em saúde do homem parece ser novidade, contudo essa temática já se faz presente em algumas discussões desde a década de 70 do século passado, principalmente, tratando-se de estudos norte-americanos. Alguns desses estudos já traziam a reflexão que o modelo hegemônico de masculinidade poderia produzir déficit dos indicadores de saúde, não só para si, mas, para a sociedade (COURTENAY, 2000; SCHRAIBER et al, 2005).

A partir da década de 90, com o advento do neoliberalismo e de outras transformações socioculturais, a representação do homem "virill" e "forte" começou a ser questionada por vários segmentos da sociedade, e, principalmente, pelo próprio homem. Dessa maneira, inicia-se uma importante discussão sobre qualidade de vida, 
impulsionando o debate do pouco cuidado que a maioria dos homens tem em relação à sua saúde.

Durante sua posse como Ministro da Saúde em março de 2007, o médico sanitarista José Gomes Temporão anunciou, dentre suas 22 prioridades de gestão, a criação da Política Nacional de Atenção Integral à Saúde do Homem (PNAISH), que só foi regulamentada em 27 de agosto de 2009 através da portaria GM n 1.944, tendo como objetivo:

(...) promover a melhoria das condições de saúde da população masculina brasileira, contribuindo, de modo efetivo, para a redução da morbidade e da mortalidade dessa população, por meio do enfrentamento racional dos fatores de risco e mediante a facilitação ao acesso (grifo nosso), às ações e aos serviços de assistência integral à saúde (BRASIL, 2009, p.65).

A PNAISH, desde seu início, foi idealizada a partir de algumas diretrizes da Política Nacional de Atenção Básica, representada hoje, através da ESF. O objetivo principal dessa política é promover ações que possam desencadear uma melhor compreensão do processo saúde-doença no universo masculino no intuito de aumentar a expectativa de vida e de reduzir os elevados indicadores da morbimortalidade nessa população (BRASIL, 2008).

Sob esta perspectiva, tais experiências podem vir a colaborar para a promoção de reflexões e debates no universo acadêmico e de serviço de saúde, visando à identificação de barreiras ainda existentes no imaginário do "ser homem". Dessa forma, espera-se uma contínua facilitação na formação de profissionais sensíveis quanto à mobilização de estratégias que estimulem uma maior demanda do público masculino ao cuidado de sua saúde.

\section{METODOLOGIA}

\subsection{CARACTERIZAÇÃO DA ÁREA DE ESTUDO}

O presente estudo foi motivado a partir da vivência na Unidade de Saúde da Família (USF) Aurélio Schiarreta na cidade de Jequié/BA através da disciplina Estágio de Docência do Programa de Pós-Graduação em Enfermagem e Saúde em nível de Mestrado Acadêmico, do Departamento de Saúde (DS) da Universidade Estadual do Sudoeste da Bahia (UESB), Campus de Jequié. 
A unidade atende às necessidades de saúde de aproximadamente 2.000 mil famílias dos bairros Itaigara I e II e Prodeccor, sendo composta de três Equipes de Saúde da Família para melhor atender à demanda.

A estrutura física da unidade compreende 01 sala de vacina, 01 sala de procedimentos, 01 sala de esterilização, 01 farmácia, 01 consultório odontológico, 03 consultórios de enfermagem, 02 consultórios médicos, 02 sanitários para os usuários, 02 sanitários para os funcionários, 01 copa e 01 recepção. Quanto ao horário de funcionamento, a USF atende os usuários das 07:30 às 12:00h e das 14:00 às 17:00h.

\subsection{VIVÊNCIA NA UNIDADE DE SAÚDE DA FAMÍLIA}

A motivação do campo para a prática docente numa USF surgiu devido ao objeto de estudo da dissertação que foi analisar a dinâmica da acessibilidade masculina à Estratégia de Saúde da Família. Nada mais oportuno e factível, então, de aproximar as inquietações enquanto pesquisador, como a de vivenciar e colaborar com a prática docente da disciplina Estágio Supervisionado I oferecida no VIII Semestre de Graduação em Enfermagem da UESB o qual utiliza a USF como espaço de produção do saber em saúde coletiva.

No Plano de Curso elaborado para a disciplina, elencou-se como objetivos a serem alcançados:

- Possibilitar ao mestrando a aproximação com o universo docente, discente, equipe de saúde e comunidade, através da docência na área de saúde coletiva.

- Instrumentalizar o mestrando para a prática docente;

- Aprofundar conceitos do processo ensino-aprendizagem na área da saúde coletiva com enfoque na Estratégia de Saúde da Família;

- Discutir a implementação de práticas de saúde a partir das diretrizes da Política Nacional de Atenção Integral a Saúde do Homem.

Para isso, fundamentou-se a prática docente a partir de alguns eixos norteadores: Processo Ensino-Aprendizagem, Estratégia de Saúde da Família e a Política Nacional de Atenção Integral a Saúde do Homem. Foram adotados procedimentos de ensino: educação em saúde na sala de espera, leitura e discussão de textos científicos sobre a saúde do homem com os discentes, planejamento e realização da "I Oficina de Saúde do 
Ensino, Saúde e Ambiente - V5 (3), pp. 84-93, dez. 2012

Homem” direcionada aos Agentes Comunitários de Saúde (ACS), e, por fim, supervisão direta aos discentes em suas atividades acadêmicas da disciplina Estágio Supervisionado I.

É importante salientar que a finalidade desta experiência acadêmica não se restringiu apenas a focalizar a saúde do homem de forma fragmentada e/ou dissociada do seu universo familiar, evitando assim que se tornasse mais uma ação voltada a um público específico na ESF. Ao contrário, pretendeu-se contextualizar a figura do homem numa perspectiva ampliada, dinâmica e transversalizada a partir do panorama em que o mesmo está inserido nas práticas do cuidado à saúde da família, constituindo o eixo estrutural preconizado pela ESF.

\section{RESULTADOS E DISCUSSÃO}

A primeira atividade desenvolvida no Estágio de Docência foi a educação em saúde com os usuários que esperavam para serem atendidos pelos profissionais da USF. Esse momento, também, conhecido como "Sala de Espera", é definido pelo Ministério da Saúde como:

(...) um processo sistemático, contínuo e permanente que objetiva a formação e o desenvolvimento da consciência crítica do cidadão, estimulando a busca de soluções coletivas para os problemas vivenciados e a sua participação real no exercício do controle social (BRASIL, 2007, p.22).

Diante deste contexto, pretendeu-se despertar a reflexão dos usuários sobre a saúde do homem, tema principal em discussão. Contou-se com a participação de um discente nesta atividade, que colaborou na intermediação das falas que iam surgindo.

Tentou-se, durante a atividade, fugir do modelo cartesiano que desconsidera a construção do conhecimento nas práticas de educação em saúde. Ao contrário, trabalhou-se numa vertente da problematização da temática no intuito de aproximar as discussões com a realidade daqueles usuários, desencadeando, assim, o despertar crítico do problema coletivo, que vem afetando de alguma maneira o cotidiano da família contemporânea brasileira.

Inicialmente, a equipe de trabalho foi apresentada e explicado o objetivo das atividades. Em seguida, propositalmente, foi questionado quantos homens estavam presentes naquele ambiente. Nesse instante, observou-se que a "invisibilidade" do homem foi percebida sob um olhar curioso, e, também, crítico. Ficaram evidentes os 
olhares de interrogação e interesse de todos à medida que se avançava nas questões disparadoras, como: a relação do homem com o cuidado a sua saúde, a pouca presença masculina nos postos de saúde, o processo de construção da masculinidade e sua influência na saúde do homem, dentre outras discussões.

Foi um momento bastante enriquecedor, onde foi visualizado que o próprio discente colaborador nesta atividade, reportou após a mesma dizendo sobre a importância daquele momento em sua prática acadêmica, visto que, até então, não tinha se atentado para as nuances que permeiam a saúde do homem e o que estas podem vir a repercutir na dinâmica familiar.

Outra atividade realizada foi a "I Oficina de Saúde do Homem". A oficina contou com a participação de 18 ACS pertencentes das três equipes de Saúde da Família da USF, além dos atores envolvidos no planejamento da mesma (mestrando, enfermeiras da USF e docentes/discentes da disciplina Estágio Supervisionado I). A opção de direcionar esta primeira oficina sobre Saúde do Homem para os ACS partiu de uma decisão coletiva baseada na híbrida importância social desse profissional na ESF.

O ACS é uma pessoa que convive com a realidade e as práticas de saúde do bairro onde mora e trabalha, e ser formado a partir de referenciais biomédicos, faz deste um ator que veicula as contradições e, ao mesmo tempo, a possibilidade de um diálogo profundo entre esses dois saberes e práticas (NUNES, 2002, p.2)

No intuito de melhor operacionalização das atividades, a oficina foi dividida em três momentos:

I) Abertura da Oficina, explicitando seus objetivos, e posterior dinâmica de aquecimento entre os participantes. Utilizou-se bolas de assopro onde em seu interior havia questionamentos disparadores sobre a temática;

II) Construção e apresentação de painéis sobre a saúde do homem pelos ACS, enfatizando o seu entendimento, os motivos que interferem na procura do homem as unidades de saúde e as estratégias para o processo de implementação da Política;

III) Apresentação e discussão da Política Nacional de Atenção Integral à Saúde do Homem, com ênfase ao papel do ACS.

Os dois primeiros momentos da Oficina foram realizados pelos discentes da disciplina ECS I. A última etapa ficou sob a responsabilidade dos professores. É importante ressaltar que esta divisão se deu a título de funcionalidade da atividade, visto que todo o processo de planejamento da oficina foi construído de forma coletiva. 
Foi utilizada a metodologia da Oficina Pedagógica como estratégia de construção do conhecimento, a partir de um enfoque problematizador, visando, assim, a aproximação do ACS com o tema discutido.

As Oficinas Pedagógicas tem sido bastante utilizadas em capacitações, principalmente, para os profissionais da área da saúde, pois além de propiciarem um ambiente acolhedor, favorecem, também, à construção coletiva, reflexiva e crítica de conhecimentos sobre determinado assunto superando a distância entre a teoria e prática, entre conhecimento e trabalho, e entre a educação e a vida (ANDER-EGG, 2000).

Essa atividade superou as expectativas diante das significativas e coerentes falas dos ACS sobre a complexidade que perpassa a relação do homem com o cuidado à saúde. Foi constatado um claro entendimento pelos mesmos quanto aos princípios da PNAISH, sobre os motivos que podem vir a interferir na procura dos homens aos serviços de saúde, e, por fim, estratégias que possam auxiliar a efetivação dessa política de saúde tanto pela comunidade quanto dos profissionais de saúde.

As atividades permitiram realizar a supervisão dos discentes nas consultas de enfermagem, em particular na atenção a saúde da criança e do adulto. Nas primeiras consultas apenas se observou como se procedia ou não a abordagem integral da assistência ao usuário como integrante de uma família, foco principal da ESF, a fim de que se pudesse, no decorrer do atendimento a outros usuários, discutir determinados posicionamentos e, ao mesmo tempo, refletir quanto a implementação de novas posturas e ações assistenciais.

Observou-se que os discentes possuem bom conteúdo técnico-científico, responsabilização, vínculo e uma comunicação efetiva para com os seus usuários assistidos nas consultas de enfermagem. Todavia, também, foi notado que há uma dificuldade quanto à promoção de um cuidado integral que perpasse às aparentes fronteiras das necessidades imediatas de saúde trazidas pelos usuários.

Por exemplo, durante as consultas de enfermagem a pessoas portadoras de Hipertensão Arterial foi possível presenciar que o discente por muitas vezes não conseguiu assistir aquele usuário além dos cuidados voltados ao seu estado de saúde a partir do diagnóstico clínico. Assim, nesse momento, perdeu-se a oportunidade de perceber esta pessoa inserida num contexto familiar e epidemiológico, necessitando de um olhar ampliado sobre o seu processo saúde-doença.

Outro momento que chamou a atenção foi a consulta de enfermagem à Saúde da Criança. Verificou-se que durante o atendimento, as crianças do sexo masculino, dentre alguns brinquedos espalhados na sala, naturalmente, pegavam a boneca e começavam 
então a brincar. Tal fato este que era prontamente repreendido pela maioria das mães que os acompanhavam. Essa atitude vinda da genitora, por vezes, única responsável pela formação de sua prole gerou várias reflexões aos que estavam presentes naquele momento, no que se refere à influência exercida pelos pais na construção da masculinidade e de que forma esta poderia implicar no processo saúde-doença do homem.

Ambas as situações foram discutidas com os discentes no decorrer das consultas de enfermagem, e no findar de cada período. Dessa maneira, era realizada a discussão de alguns artigos científicos referentes a diversos contextos que perpassam a saúde do homem na atualidade.

Observou-se que os discentes demonstraram interesse quanto aos assuntos discutidos, em especial sobre a saúde masculina. Visualizou-se que a partir desta experiência, os discentes puderam refletir sobre o seu processo de trabalho, o que, naturalmente, desencadeou a implementação de algumas ações em suas práticas assistenciais, e, por conseguinte, a adoção de novas posturas acadêmicas na Unidade de Saúde da Família.

\section{CONSIDERAÇÕES FINAIS}

Acredita-se que esta experiência tenha despertado em todos os envolvidos (mestrando, docentes, discentes, enfermeiras e outros profissionais da USF, ACS e comunidade) a importância da discussão da saúde do homem na ESF. Não obstante a isso, é incontestável registrar a valorosa participação dos discentes da disciplina ECS I na construção desses novos olhares, sendo possível vivenciar o processo ensinoaprendizagem de forma crítica, motivadora e prazerosa.

O número reduzido de discentes, apenas quatro, constituiu um fator positivo no transcorrer das atividades, pois permitiu uma maior aproximação e interação da relação docente/discente durante o processo ensino-aprendizagem, até mesmo por conta da reduzida carga horária da disciplina estágio de docência $(30 \mathrm{H})$, quando comparada a disciplina ECS I (270H).

É importante ressaltar que as atividades desenvolvidas durante essa vivência acadêmica direcionadas ao público masculino foram construídas e planejadas de forma coletiva e dinâmica entre os sujeitos envolvidos. Espera-se que este estudo possa subsidiar novas experiências que permitam a reflexão da saúde do homem na ESF, visando assim uma melhor compreensão coletiva das diversas partes envolvidas. 


\section{REFERÊNCIAS}

ANDER-EGG A.S. In: Omiste et al. Formação de grupos populares: uma proposta educativa. Rio de Janeiro: DP\&A; 2000.

BANKS, I. New models for providing men with health care. Editorial. Journal of Men's Health and Gender, v.2-3, n.1, p.155-158, 2004.

BRASIL. Ministério da Saúde. Secretaria de Assistência à Saúde Coordenação de Saúde da Comunidade. Saúde da Família: Uma Estratégia para a reorientação do modelo assistencial. Brasília-DF: Ministério da Saúde, 1997.

Ministério da Saúde. Diretrizes de educação em saúde visando à promoção da saúde: documento base - documento I/Fundação Nacional de Saúde - Brasília: Funasa, $2007.70 \mathrm{p}$.

Ministério da Saúde. Secretaria de Atenção à Saúde. Departamento de Ações Programáticas Estratégicas - Política Nacional de Atenção Integral à Saúde do Homem. Brasília-DF: Ministério da Saúde, 2008, 46p.

Portaria $n^{\circ} 1.944$ de 27 de agosto de 2009. Institui no âmbito do Sistema Único de Saúde (SUS), a Política Nacional de Atenção Integral à Saúde do Homem. Diário Oficial da União [da República Federativa do Brasil], Brasília, n.165, p.61, 27 de agosto de 2009, Seção 1

COURTENAY, W. H. Constructions of masculinity and their influence on men's wellbeing: a theory of gender and health. Social Science \& Medicine, v.50, n.10, p.1385$1401,2000$.

IBGE. Instituto Brasileiro de Geografia e Estatística. Censo 2010. Disponível em: <http://www.ibge.gov.br/cidadesat>. Acesso em: 21 de outubro de 2012.

NUNES, M. O. et al. O agente comunitário de saúde: construção da identidade desse personagem híbrido e polifônico. Cad. Saúde Pública, Rio de Janeiro, n.18, v.6, p.1639-1646, 2002.

SCHRAIBER, L. B.; GOMES, R.; COUTO, M.T. Homens na pauta da saúde coletiva. Ciência \& Saúde Coletiva, Rio de Janeiro, v.10, n.1, p.7-17, 2005. 